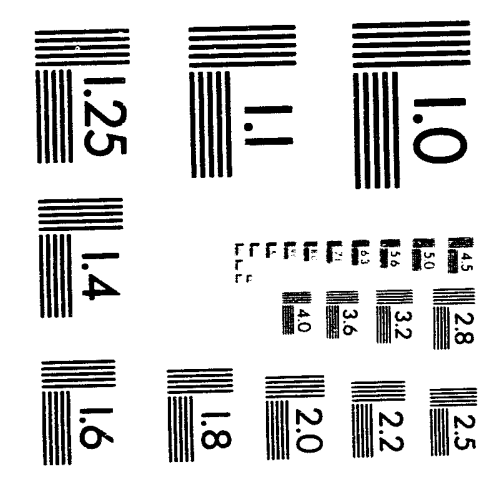



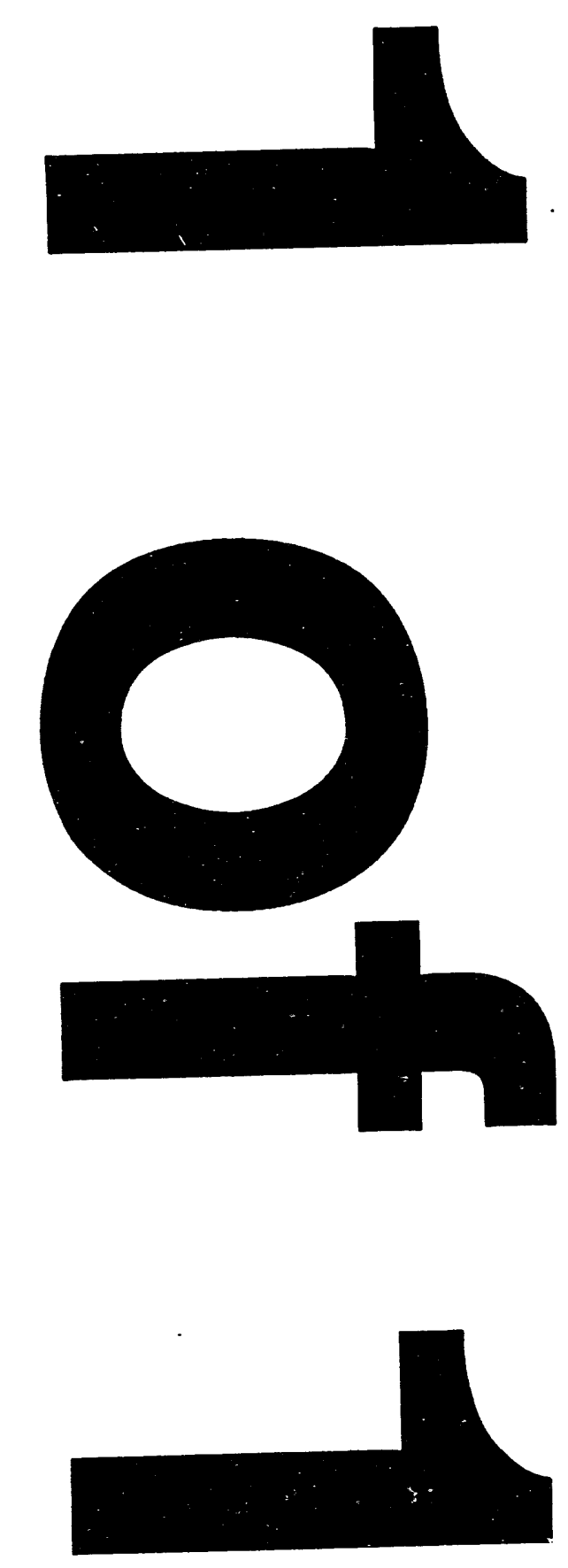
LBL-34782

\title{
THE RELATIVISTIC TREATMENT OF SYM- METRIC AND ASYMMETRIC NUCLEAR MATTER
}

\author{
Hans Huber and Manfr d K. Weigel \\ Ludwig-Maximilians-Universität München, Sektion Physik, Am \\ Coulombwall 1, D-85748 Garching, FRG \\ Fridolin Weber \\ Nuclear Science Division, Lawrence Berkeley Laboratory, Uni- \\ versity of California, Berkeley, CA 94720, U.S.A.
}

November 4, 1993

Presented at the

NATO Advanced Study Institute

HOT AND DENSE NUCLEAR MATTER

Bodrum, Turkey September 26 - October 9, 1993

To be published by Plenum Press in the Physics Series

This work was supported by the Director, Office of Energy Research, Office of High Energy and Nuclear Physics, Division of Nuclear Physics, of the U.S. Department of Energy under Contract DE-AC03-76SF00098. 


\title{
THE RELATIVISTIC TREATMENT OF SYM- METRIC AND ASYMMETRIC NUCLEAR MATTER
}

\author{
Hans Huber and Manfred K. Weigel \\ Ludwig-Maximilians-Universität München, Sektion Physik, Am \\ Coulombwall 1, D-85748 Garching, FRG \\ Fridolin Weber \\ Nuclear Science Division, Lawrence Berkeley Laboratory, Uni- \\ versity of California, Berkeley, CA 94720, U.S.A.
}

\section{INTRODUCTION}

In the last decades it was realized that certain features of the nuclear problem. can only be described by going beyond the nonrelativistic approach. Relativistic treatments of the nuclear many-body problem have advantages in several resperts, for instance $^{1,2}$ : An extremely useful Dirac phenomenology in the description of nv leortnucleus scattering ${ }^{3}$; the shift of the equilibrium density from the so-ca' ${ }^{2}$ ed "Coester band" towards the "experimental" value via a new saturation mecha us ${ }^{4}, 5$; th: natural incorporation of the spin-orbit force ${ }^{1,2}$ and the successfi! terrifition 0 . finite nuclei ${ }^{6,7}$, etc.

Naturally, there is a great desire to explore the relativistic many uody forntum

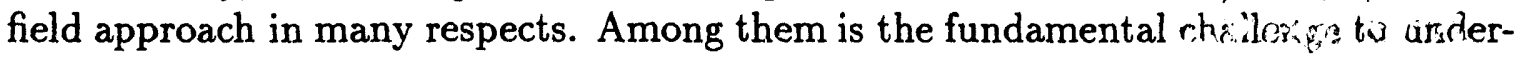
stand the properties of nuclei in terms of the interactions between its car tituents. A reliable microscopic calculation of the equation of state would be a give banes for

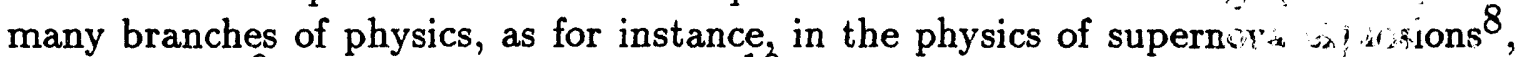
neutron stars 9 and heavy-ion scattering ${ }^{10}$. One of the basic attempis an this direction is the relativistic treatment of symmetric and asymmetric nuclear matter in many-body approximations with dynamical two-body correlations with modern oneboson-exchange potentials adjusted to the two-nucleon problem. In the next sections we are going to address this problem in the frame of the Green's function approach 
and discuss some problems as, for instance, consistence questions, predictive power, limitations etc.

\section{GENERAL THEORY}

In the model the forces between the nucleons are mediated by the exchange of mesons, hence the dynamics of the particle is governed by a Lagrangian density of the following form $1,2,4,5,11-15$ :

$$
\mathcal{L}=L_{N}+\Sigma_{M}\left(L_{M}+L_{M}^{\prime}\right)
$$

$\mathcal{L}_{N}$ denotes the Lagrangian density of noninteracting nucleons; similary, $\mathcal{L}_{M}$ describes the different free meson fields, which interact via $\mathcal{L}_{M}^{\prime}$ with the nucleons.

A suitable tool for the treatment of many-body systems is the Green's function scheme. The formulation of the problem in ladder-type approximation is an established procedure and resembles in its formal structure closely to the nonrelativistic treatment 16,17 . One obtains a coupled system of the Dyson equations for the $G$ function and the effective scattering matrix $T$ in matter

$$
\begin{aligned}
& \left\{\left(G^{0}\right)^{-1}(1,2)-\Sigma(1,2)\right\} G\left(2,1^{\prime}\right)=\delta\left(1,1^{\prime}\right) \\
& <12|T| 1^{\prime} 2^{\prime}>=<12|V| 1^{\prime} 2^{\prime}-2^{\prime} 1^{\prime}>+<12|V| 34>\Lambda(34,56)<56|T| 1^{\prime} 2^{\prime}>
\end{aligned}
$$

We employ the convention to sum or to integrate over all doubly occuring variables. Here $V$ denotes the OBE-potential

$$
\left.<12|V| 1^{\prime} 2^{\prime}\right\rangle=\sum_{M=(\sigma, \omega, \ldots)}<12\left|V_{M}\right| 1^{\prime} 2^{\prime}>
$$

and the self-energy is given by

$$
\Sigma(1,2)=-i<14|T| 52>G(5,4)
$$

The H- or HF-approximation are defined by $T=V\left(V_{A S}\right)$, respectively.

For the intermediate p-p-propagator a standard choice is the Brueckner propagator (cf. Refs. ${ }^{4}, 5,11-13,15$ ); but one also use the so-called $\Lambda$-approximations ${ }^{13}, 14$, defined as $\left(G^{0}\right.$ denotes the free propagator; $\left.i j=00,01,11\right)$ :

$$
-i \Lambda^{i j}(12,34)=\left\{\begin{array}{l}
G^{0}(1,3) G^{0}(2,4) \\
\frac{1}{2}\left(G^{0}(1,3) G(2,4)+G(1,3) G^{0}(2,4)\right) \\
G(1,3) G(2,4)
\end{array}\right.
$$

which are obtained from the Martin-Schwinger approximation scheme ${ }^{18}$ by taking dynamical correlations into account, which are connected with the potential (for instance, $<12|V| 34>G\left(34,1^{\prime} 2^{\prime}\right)$ is included but $<12|V| 34>G\left(1^{\prime} 4,32^{\prime}\right)$ is replaced by $<12|V| 34>\left(G\left(1^{\prime}, 3\right) G\left(4,2^{\prime}\right)-G\left(1^{\prime}, 2^{\prime}\right) G(4,3)\right)$, for more details and a comparison between the different approximations, see Refs. $13,17,18,19,20$. It turns out that 
all relativistic approximations give a shift towards the semiempirical values and the RBHF-results are located between the $\Lambda^{00}$ - and the $\Lambda^{01}$-results $\left(\Lambda^{00}\right.$ gives the lowest values for $E / A^{13}$; for the treatment of the full ladder approximation see Ref. ${ }^{14}$ ).

A useful simplification can be achieved by utilizing the spectral representation of $G$, i.e.

$$
G(p)=\int d w \frac{A(\vec{p}, \omega)}{\left(p_{0}-\mu\right)(1+i \eta)-\omega}
$$

since all desired quantities can be determined by the self-energy $\Sigma$ and the spectral function $A$ alone ${ }^{13}, 17$.

\section{DISCUSSION}

Despite the formal similarity to the nonrelativistic case the relativistic situation is much more complicated; due to, for instance:

1. Energy-dependent meson-potentials (retardation)

2. Bethe-Salpeter equation in four dimensions

3. Dirac algebra ( $T$-matrix has in principle 256 elements with respect to spin); $\Sigma$ (and $A$ ) has scalar, vector and time-like contributions with the following structure:

$$
\Sigma(p)=\Sigma_{s}(p)+\Sigma_{v}(p)(\hat{p} \cdot \vec{\gamma})+\gamma^{0} \Sigma_{0}(p)
$$

4. Self-consistent single-particle basis (spinors) are not a priori known; therefore the solution in the self-consistent basis in the full Dirac space is rather complicated $^{13}, 15$.

First of all one needs self-consistent spinors, since the relativistic saturation mechanism depends strongly on the lower parts of the spinors, and give a decreasing (increasing) contribution for the $\sigma-(\omega-)$ mesons with increasing density. This feature leads to a non-monotonic behaviour of the kinetic and potential part of the energy. If one uses free spinors one gets back the nonrelativistic features (see Fig. 1).

The consistency problem whether a quasi-particle picture is applicable depends on the energy-dependence of the self-energy. For obtaining a single-particle energymomentum relation it is necessary, that $\left|\frac{\partial \Sigma}{\partial \omega}\right|$ is smaller than 0.5 . This question is treated in more detail in Refs. 13,17 . For instance, the momentum baryon distribution in the relativistic case is given by:

$$
\varrho_{B}(\vec{p})=\left\{\frac{W(p)}{\left|W-\left[m^{*} \frac{\partial \Sigma_{v}}{\partial \omega}+\tilde{k} \frac{\partial \Sigma_{v}}{\partial \omega}+W \frac{\partial \Sigma_{0}}{\partial \omega}\right]\right|}\right\}_{p_{0}=\omega(\tilde{p})},
$$




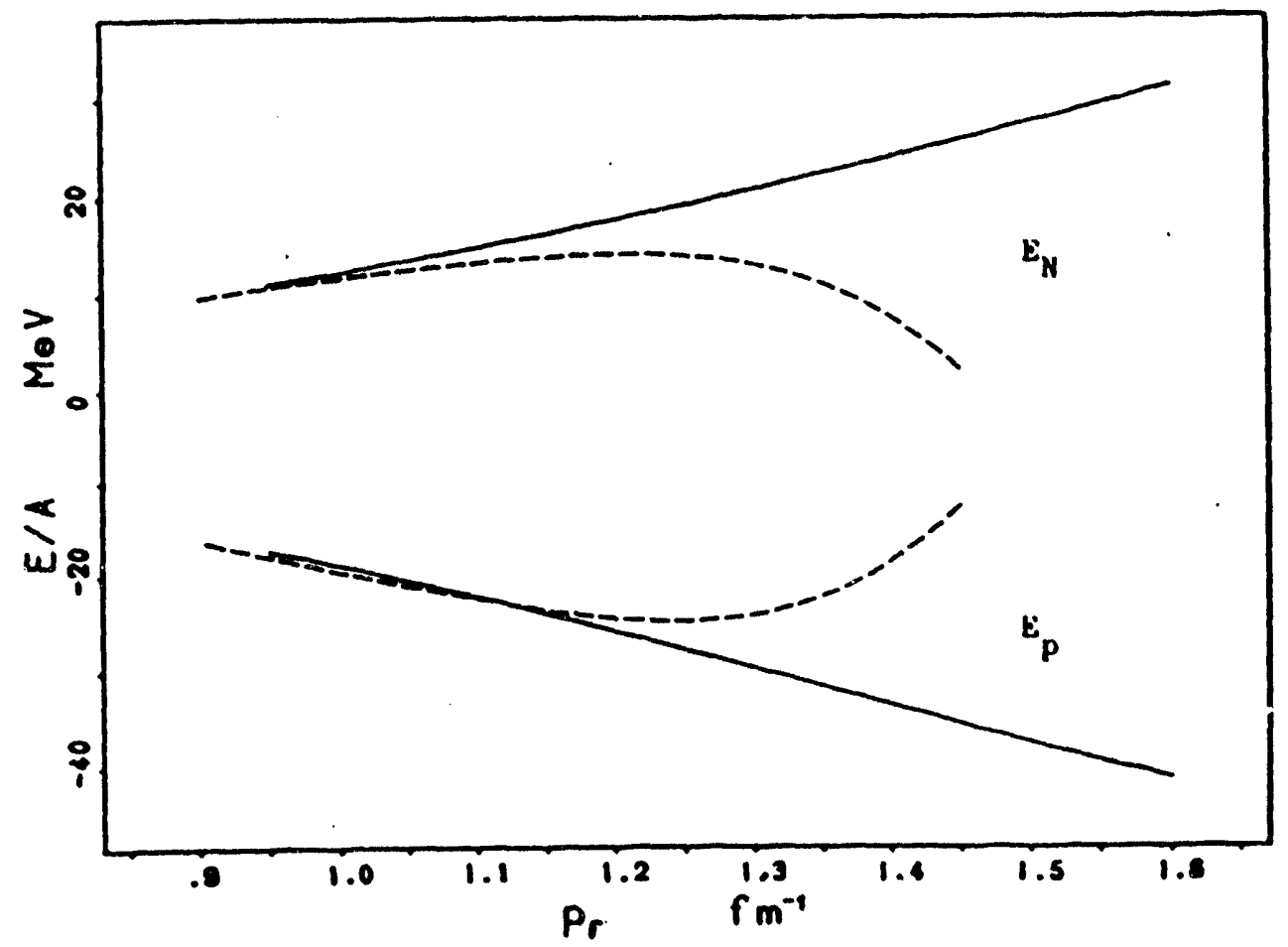

Figure 1. Illust:ation of the influence of the self-consistent basis (relativistic saturation mechanism): Kinetic (Dirac) and potential energy in RBHF-approximation for the OBE-potential Ho2 ${ }^{13}$. The dotted curves correspond to the self-consistent basis (i.e. non-monotonic behaviour); the solid curves give the outcome with free spinors (i.e. similiar to the non-relativistic treatment).

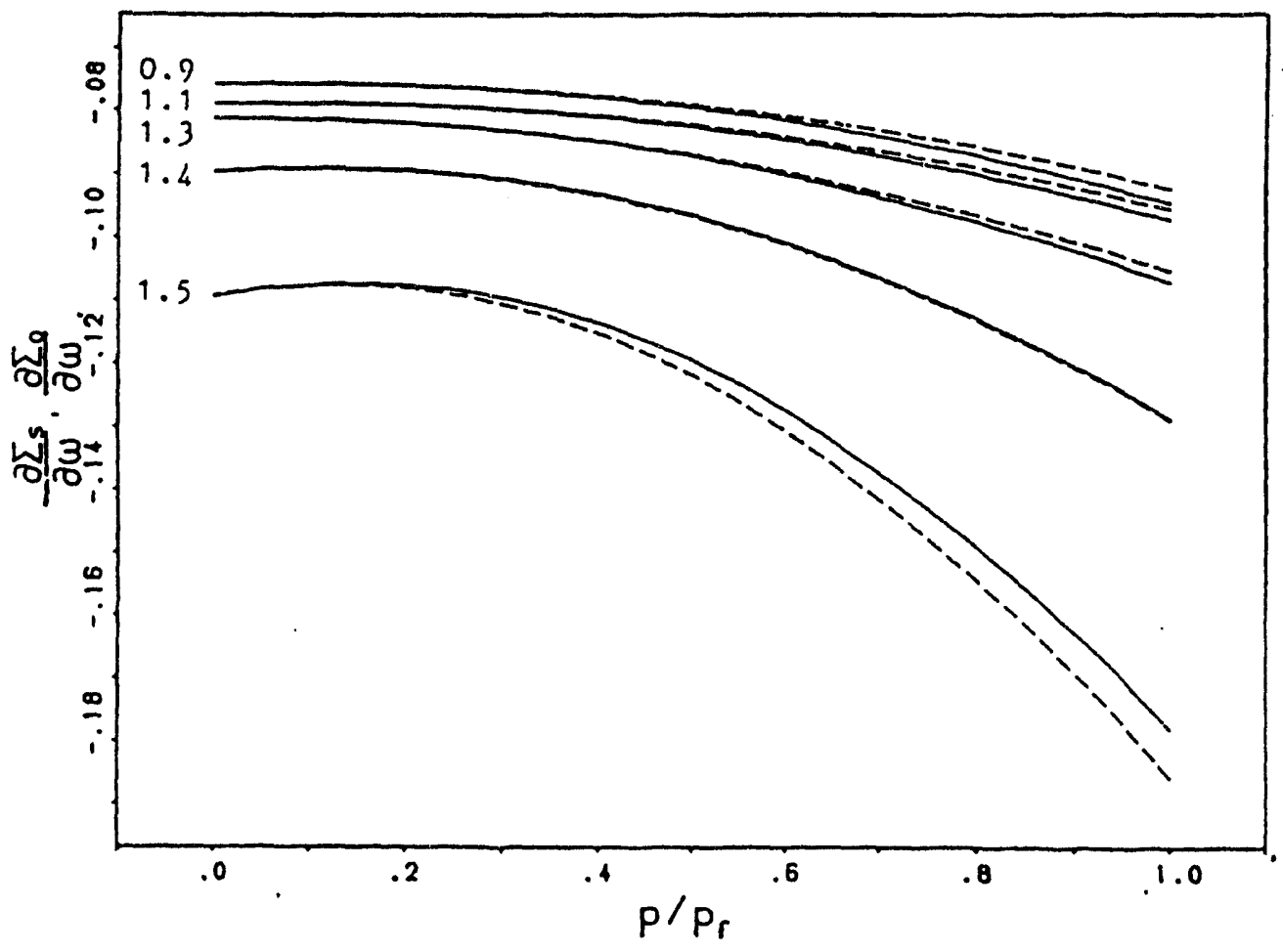

Figure 2. Energy derivatives $\frac{\partial \Sigma_{S}}{\partial \omega}$ (solid curves) and $-\frac{\partial \Sigma_{0}}{\partial \omega}$ (dashed curves) vs. $p / p_{F}$ for different Fermi momenta in $\Lambda^{00}$ - approximation (OBE-potential Ho2 ${ }^{13}$ ). 
Table 1: Saturation properties of nuclear matter in different approximations (Brockmann potential $\mathrm{B}^{5}$ ): $\mathrm{RBHF}^{(1)}$ (full basis; momentum dependent self-energy), $\mathrm{RBHF}^{(2)}$ (full basis; momentum averaged self-energy), $\mathrm{RBHF}^{(3)}$ (positive spinors only; momentum independent self-energy $\left.{ }^{5}\right) ; \Lambda^{00}\left(\Lambda^{00}\right.$ - approximation). For comparison we also give the outcome of the relativistic HF-approximation, where $\rho$ and $E / A$ are adjusted ${ }^{22}: \mathrm{RHF}^{(1)}\left(\sigma-, \omega-\right.$ mesons only), $\mathrm{RHF}^{(2)}(\sigma-, \omega-, \pi-$ and $\rho-$ mesons; $\left.f_{\rho} / g_{\rho}=6.6\right), \operatorname{RHF}^{(3)}\left(\sigma-, \omega-, \pi-\right.$ and $\rho-$ mesons; $\left.f_{\rho} / g_{\rho}=3.7\right)$.

\begin{tabular}{ccccc}
\hline Method & $\begin{array}{c}\mathrm{E} / \mathrm{A} \\
{[\mathrm{MeV}]}\end{array}$ & $\begin{array}{c}\rho \\
{\left[\mathrm{fm}^{-3}\right]}\end{array}$ & $\begin{array}{c}\mathrm{K} \\
{[\mathrm{MeV}]}\end{array}$ & $\begin{array}{c}a_{4} \\
{[\mathrm{MeV}]}\end{array}$ \\
\hline $\mathrm{RBHF}^{(1)}$ & -14.8 & 0.170 & 263.7 & 33.9 \\
$\mathrm{RBHF}^{(2)}$ & -15.7 & 0.172 & 248.9 & 32.8 \\
$\mathrm{RBHF}^{(3)}$ & -13.6 & 0.174 & 249.0 & \\
$\Lambda^{00}$ & -21.9 & 0.210 & 259.6 & 33.8 \\
$\mathrm{RHF}(1)$ & -15.75 & 0.148 & 610.0 & 28.9 \\
$\mathrm{RHF}^{(2)}$ & -15.75 & 0.148 & 360.0 & 43.3 \\
$\mathrm{RHF}^{(3)}$ & -15.75 & 0.148 & 460.0 & 38.6 \\
\hline
\end{tabular}

which reduces to the step function for $\frac{\partial \Sigma}{\partial \omega}=0$. It turns out that the energydependence is sufficiently weak for the applicability of the single-particle description (see Fig. 2).

In the pioneering work of the Brooklyn group ${ }^{11}$ the problem was treated in the full Dirac space but the relativistic effect was only included in first-order perturbation theory, so avoiding the complicated self-consistency problem. Therefore a comparison with other treatments is rather difficult.

Due to the complexity of the problem one has tried in most treatments to avoid the solution in the full Dirac space.

The standard method, applied in Refs. ${ }^{4}, 5,12,14$, makes a non-unique ansatz for the $T$-matrix in terms of five independent Fermi invariants (in Refs. ${ }^{4}, 14$ the pseudoscalar invariant is replaced by the pseudovector invariant)

$$
T=T^{S} I^{(1)} I^{(2)}+T^{\nu} \gamma_{\mu}^{(1)} \gamma^{(2) \mu}+T^{T} \tau_{\mu \nu}^{(1)} \tau^{(2) \mu \nu}+T^{P S} \gamma_{5}^{(1)} \gamma_{5}^{(2)}+T^{A} \gamma_{5}^{(1)} \gamma_{\mu}^{(1)} \gamma_{5}^{(2)} \gamma^{(2) \mu}
$$

and obtains the solution in the c.v. frame for positive spinors only. Afterwards they transform the $T$-matrix into the nuclear matter frame. Once a specific value of $m^{*}$ is chosen and Lorentz boosting mixes only positive-energy helicity spinors themselves one determines only the positive-energy matrix elements. For that reason the full matrix structure of $T$, and hence of $\Sigma$, is not uniquely determined. It was shown that the results for $\Sigma$ depend on the chosen decomposition 20 .

The other method, used by Brockmann and Machleidt ${ }^{5}$, avoids this procedure by the assumption that the scalar and time-like parts of $\Sigma$ are momentum independent and approximate the positive-energy matrix elements of $\Sigma$, obtained from the RBHsolution via

$$
<\phi|\Sigma| \phi>=\frac{m^{*}}{E^{*}} \Sigma_{S}+\Sigma_{0}
$$


Both approaches have been discussed and critized in more detail in Ref. ${ }^{21}$. Despite the weak momentum dependence of $\Sigma_{0}$ for the whole range of $m^{*}\left(0.5-1.0 m_{N}\right)$ the absolute values for $\Sigma_{0}$ differ strongly $(393-(-117) \mathrm{MeV})^{21}$ and there is no a priori reason to prefer a $m^{*}$-value with the smallest deviation from a constant; or otherwise expressed a direct calculation of $m^{*}\left(p_{F}\right)$ is necessary.

\section{RESULTS}

In order to clarify the situation we have calculated for a modern OBE-potential (Brockmann $B)^{5}$ the properties of nuclear and asymmetric matter in the full Dirac space. The results with comparison between the different approaches are shown in Table 1. It turns out that, at least for the chosen potential, the differences between the full Dirac space results and the Brockmann treatment are not very large. The $\Lambda^{00}$-approximation gives, as expected, a higher density and energy, respectively. Also the bulk symmetry energy $a_{4}$ agrees reasonable with the semiempirical value. The phenomenological HF-treatment, it seems, is not capable to reproduce the incompressibility $K$ and $a_{4}{ }^{22}$. (A further increase in the $\rho$-meson tensor coupling $f_{\rho} / g_{\rho}$ would decrease $K$ ). In Figs. 3 and 4 we show the EOS of state in the RBHF- and $\Lambda^{00}$. approximation for different aysmmetry parameters $\delta=\left(\rho_{n}-\rho_{p}\right) / \rho$. Furthermore we tested the validity of the quadratic dependence of the energy upon the asymmetry parameter $\delta$. Our results confirm this empirical law also for higher values of $\delta$ (see Fig. 5).

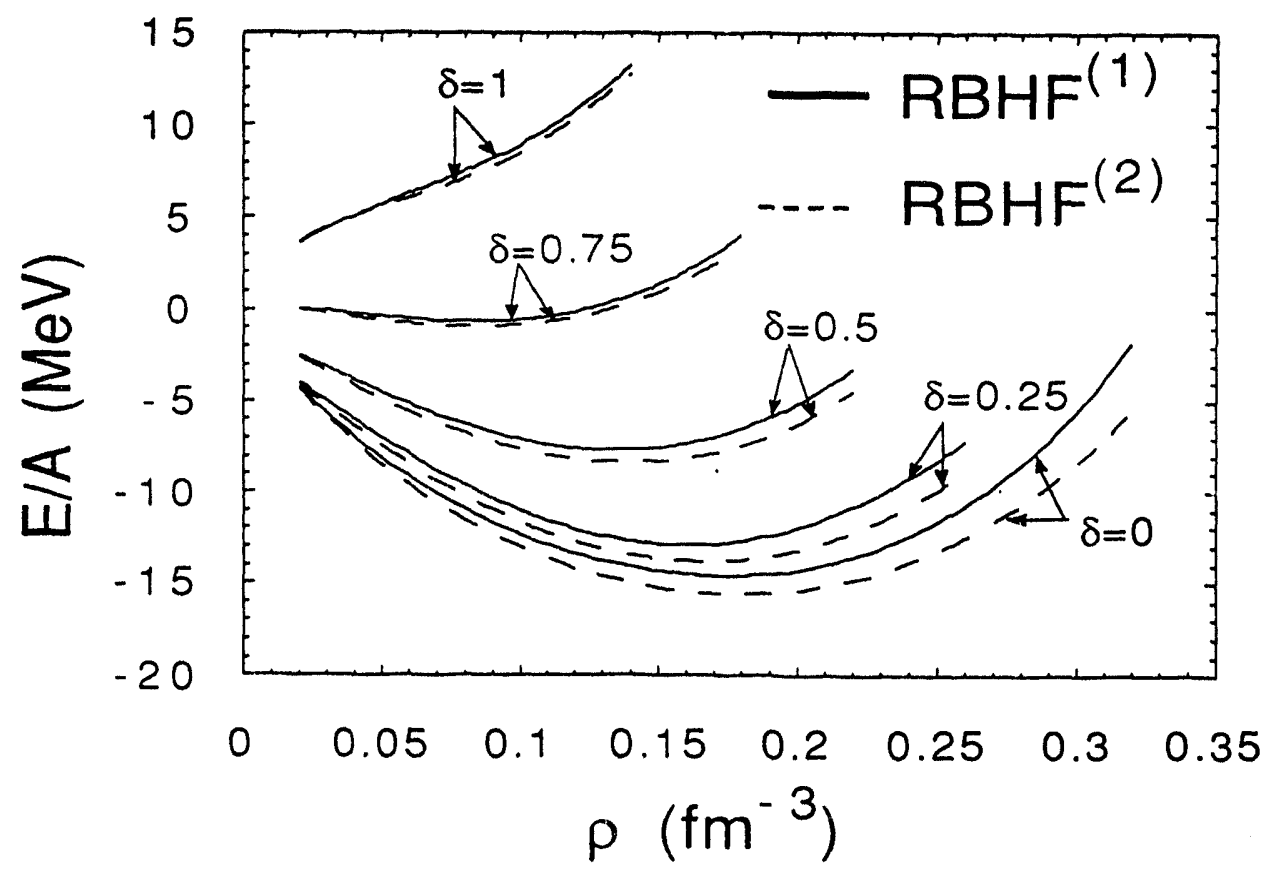

Figure 3. Binding energy per nucleon versus density for different asymmetries in the RBHFapproximation (Brockmann potential $\mathrm{B}^{5}$ ). The solid (dashed) curves correspond to the treatment with (or averaged) momentum dependency of the self-energies. 


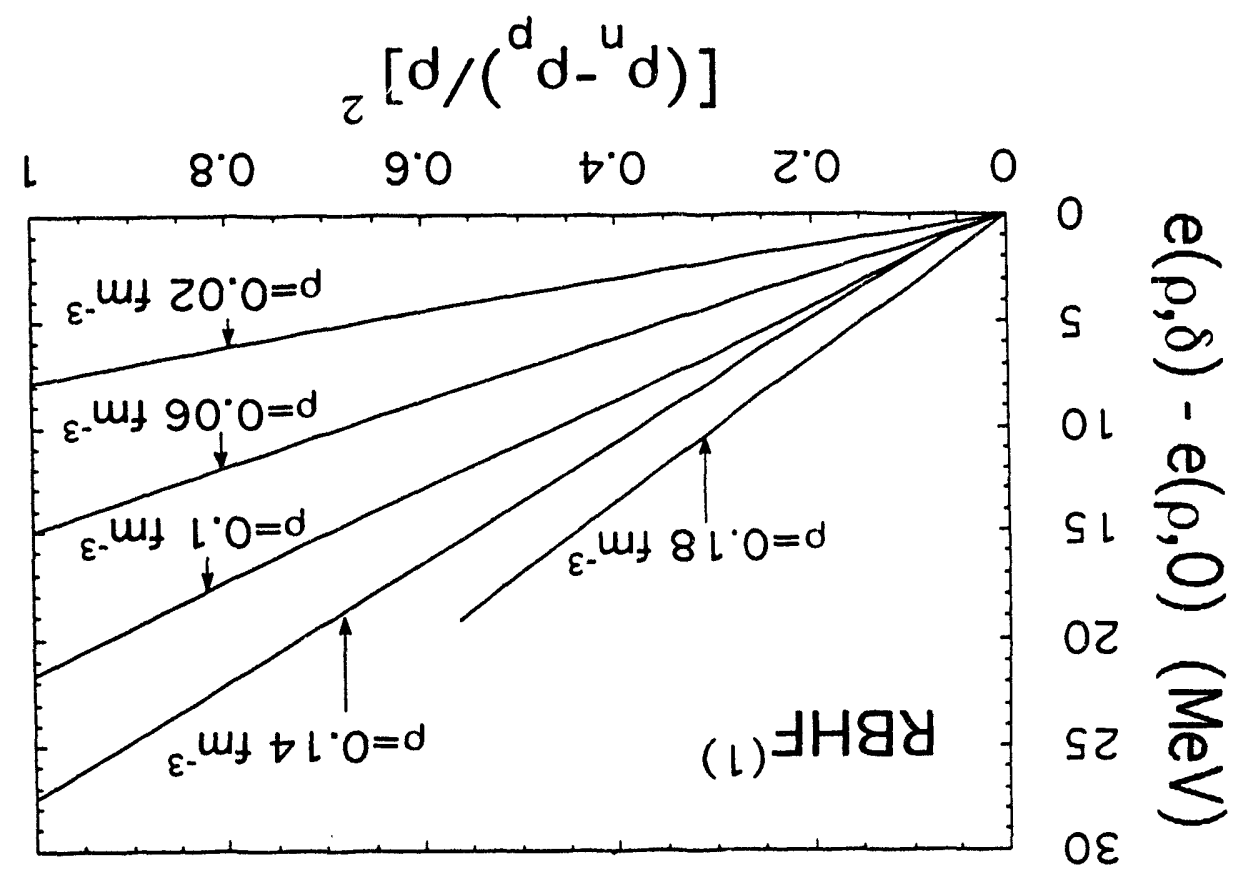

uoțeựxoddde- $00 \mathrm{~V}$

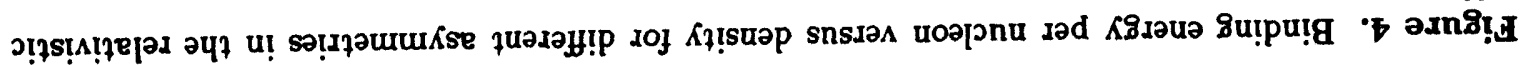

$$
\left(\varepsilon-w_{f}\right) \mathrm{d}
$$

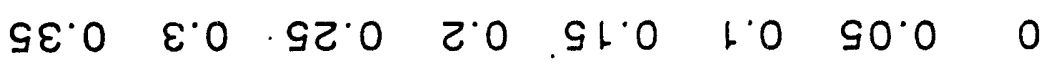

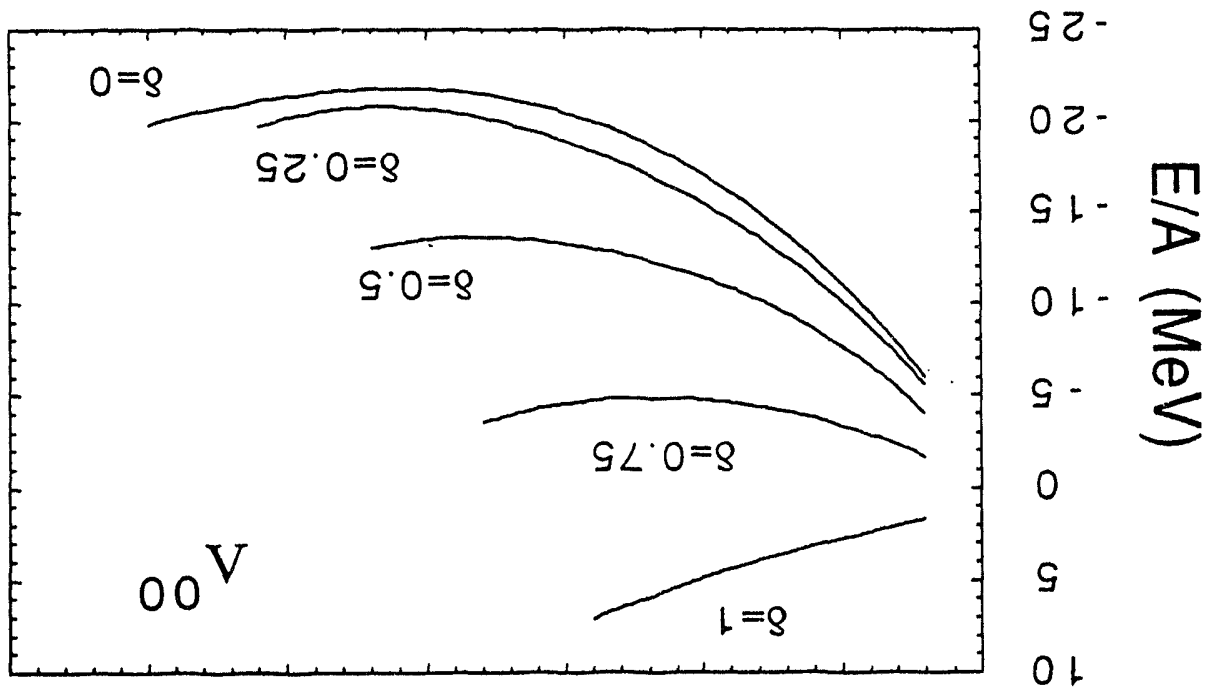


In conclusion, we have investigated the properties of symmetric and antisymmetric nuclear matter by solving self-consistently the relativistic problem with dynamical two-body correlations for a modern OBE-potential in the full Dirac space. It seems that the case of momentum averaged self-energies is applicable for densities equal or below the nuclear matter equilibrium density but for higher densities one should include the momentum-dependence of the self-energy.

\section{SUMMARY}

In the framework of relativistic nuclear field theory we discuss and compare the different approaches in the treatment of the nuclear-many-problem with inclusion of two-body correlations. The equations are solved self-consistently in the full Dirac space, so avoiding the ambiguities in the choice of the effective scattering amplitude. The results are compared with the standard method, where one only determines the scattering amplitude for positive energy spinors. Furthermore we tested the assumption of momentum independent self-energy. The results for asymmetric matter are in the structure similiar to the outcome of the relativistic Hartree-Fock approximation, but differ from the nonrelativistic treatment. The agreement with the empirical values is quite satisfactory.

\section{REFERENCES}

1. B. D. Serot and J. D. Walecka, Adv. Nucl. Phys. 16 (1986) 1, and references contained therein.

2. L. S. Celenza and C. M. Shakin, Relativistic Nuclear Physics (World Scientific, Singapore 1986), and references contained therein.

3. E. D. Cooper, S. Hama, B. C. Clark and R. L. Mercer, Phys. Rev. C47 (1993) 297 , and references contained therein.

4. J. B. ter Haar and R. Malfliet, Phys. Rep. 149 (1987) 209.

5. R. Brockmann and R. Machleidt, Phys. Rev. C42 (1990) 1964.

6. P. G. Reinhard, Rep. Prog. Phys. 52 (1989) 439.

7. Y. K. Gambhir, P. Ring and A. Thimet, Ann. of Phys. 189 (1990) 132.

8. G. E. Brown, Phys. Rep. 163 (1988) 1, and references contained therein.

9. F. Weber and N. K. Glendenning, Hadronic Matter and Rotating Relativistic Neutron Stars, Proceedings of the Nankai Summer School "Astrophysics and Neutrino Physics", p. 64 - 183, Tianjin, China, 17-27 June 1991, Eds. D. H. Feng, G. Z. He, and X. Q. Li, World Scientific, 1993, and references contained therein.

10. P. Stock, Phys. Rep. 135 (1986) 259, and references contained therein.

11. M. R. Anastasio, L. S. Celenza and C. M. Shakin, Phys. Rev. C23 (1981) 2258 and 2273 . 
12. C. J. Horowitz and B. D. Serot, Nucl. Phys. A 464 (1987) 613.

13. P. Poschenrieder and M. K. Weigel, Phys. Lett. B 200 (1988) 231; Phys. Rev. C38 (1988) 471.

14. F. de Jong and R. Malfliet, Phys. Rev. C44 (1991) 998.

15. A. Amorin and J. A. Tjon, Phys. Rev. Lett. 68 (1992) 772.

16. K. A. Brueckner and C. A. Levinson, Phys. Rev. 97 (1955) 1344.

17. M. Weigel and G. Wegmann, Fortschritte der Physik 19 (1971) 451, and references contained therein.

18. P. C. Martin and J. Schwinger, Phys. Rev. 115 (1959) 1342.

19. R. Puff, A. S. Reiner and L. Wilets, Phys. Rev. 149 (1968) 778.

20. R. Machleidt, K. Holinde and Ch. Elster, Phys. Rep. 149 (1987) 1.

21. C. Nuppenau, Y. J. Lee and A. D. MacKellar, Nuc. Phys. A504 (1989) 839.

22. M. Lopez-Quelle et al., Nucl. Phys. A 483 (1988) 479. 

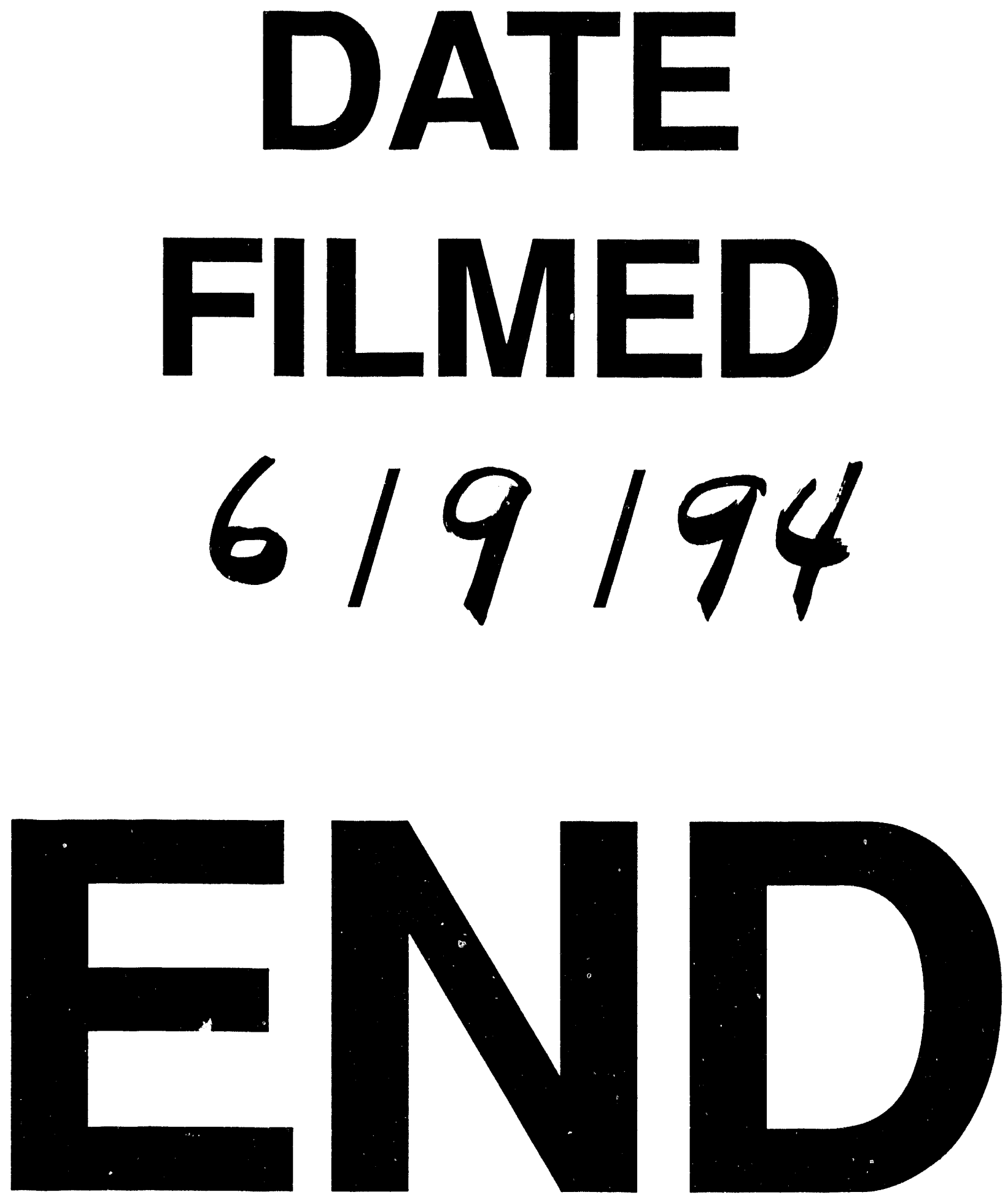